\title{
Effects of Diffraction Propagation at 24GHz Spectrum Band
}

\author{
${ }^{1}$ Femi-Jemilohun O.J and ${ }^{2}$ Walker S.D \\ ${ }^{1}$ Ekiti State University, Ado Ekiti, Nigeria; \\ ${ }^{2}$ School of Computer Science and Electronic Engineering, University of Essex, United Kingdom. \\ dunnifj@gmail.com; stuwal@essex.ac.uk
}

\begin{abstract}
The experimental measurement results of fast and slow signal fading due to corner diffraction characteristics of newly opened $100 \mathrm{MHz}$ bandwidths in the $24 \mathrm{GHz}$ spectrum for wireless communication is presented in this work. Two distinct regions are noticeable in the curve depicting these characteristics; a linear curve for the diffraction angles above 5 degrees, and a logarithmic curve for diffraction angles below 5 degrees. This feature being in agreement with what other research findings in similar working environment but at lower spectrum, as well as specifications by fundamental theory, points out the tremendous potential of this unlicensed $100 \mathrm{MHz}$ bandwidth for communication system for high and efficient delivery capacity especially in non-line of sight and obstructed transmission.
\end{abstract}

Keywords: Diffraction Propagation; slow and fast fading; Millimeter Waves; Non-Line-of-Sight; Exponential growth.

\section{Introduction}

The exponential growth in devices that require gigabits data rates application has taken over the classical microwave frequencies. This is heralded by the millimeter wave bands with huge unlicensed bandwidth capable of few gigabits data rates with high spatial reuse. The millimeter wave at $60 \mathrm{GHz}$ was the first candidate to provide such requirement for the gigabit multimedia application services, nevertheless, the atmospheric conditions effect on this band has confined it to strictly LoS propagation and a small space environment. A great opportunity and way out from this technical challenge was discovered in the newly opened $100 \mathrm{MHz}$ bandwidth window in the $24 \mathrm{GHz}$ band allocated for unlicensed usage in the wireless communication. This band has be found to perform acceptably in the NLoS propagations such as hallway and corridors as well as across offices in modern building [1, 2]. It is technologically unfeasible to achieve LoS propagation to all wireless service users, therefore, optimization and effective utilization of system capacity requires reaching many users as much as possible hence means of adequate signal transmission in the Non-line of sight nodes are crucial. Two major propagation techniques for sufficient wireless network services to consumers outside the LoS of the propagating antennas are diffraction and reflection. Among the two, the latter is a better means (reduced attenuation) for received signal prediction at any given time, especially when the diffracting angle is small. The measurement in this work evaluates the additional attenuation when $24 \mathrm{GHz}$ radio wave bends around an object 
Femi-J emilohun O.J and Walker S.D, Effects of Diffraction Propagation at 24GHz Spectrum Band, Transactions on Networks and Communications, Volume 3 No. 6, December (2015); pp: 59-64

Diffraction occurs at the edge of an impenetrable body that is large compared to wavelength of radio wave. In addition to reflection and multipath propagation that assist the signal transmission in the indoor environment, diffraction propagation results in wave propagation in the geometrical shadow region behind obstacles. It is an important propagation mechanism to be addressed as the effect of such may cause a tangible multipath propagation under both LOS and NLoS. A linear equation describes the diffraction curve with the angle of diffraction greater than 5 degrees while a logarithmic equation describes that of angle less than 5 degrees [3] [4].

\section{Fast and Slow/ Small and Large Scale Fading}

The prominent applications of millimeter wave in the NLoS environments results in the fading of the received power which are classified as small and large scale fading represented as:

$$
P_{\text {inst }}(d)=P_{\text {large }}(d)+P_{\text {small }}(d)(\mathrm{dB})
$$

Where the large scale fading determines the average characteristics of the channel due to free space path loss and the shadowing effects of large object and the small scale fading estimates the signal change in a local area of a few wavelength distance. The large scale fading is characterized by log-distance model as follows:

$$
P L_{d B}=U L_{d_{0}}+10 n \log \left(\frac{d}{d_{0}}\right)+X_{\sigma}
$$

Where $P L_{d B}$ is the average pathloss between transmission links, $U L_{d_{0}}$ is the reference path loss at $\mathrm{d}_{0}=1 \mathrm{~m}$ for indoor propagation, $\mathrm{n}$ is the path loss exponent and $\mathrm{d}$ is the separation between transmitter and receiver in meters, $X_{\sigma}$ is a zero-mean Gaussian randomly distributed variable with standard deviation $\sigma$.

One of the major causes of small scale fading is the interaction of the complex impulse-response details acquired within a limited bandwidth. Doppler spread shows that the coherence can lead to the transmission channel variation over time due to motion of objects or persons in the propagation environment or movement of the transmission links which will result in spectrum broadening. The coherence time is given by:

$$
T_{\text {Coh }}=\frac{1}{2 f_{D}}
$$

The presence of directional antenna in the propagation devices used in this experiment minimized this fading contribution by its spatial filtering feature and increase the coherence time [5]

\section{Diffraction Signal Propagation Enhancement}

"Research works have mostly focused on reflection and transmission characteristics of various building materials at millimeter waves and have largely neglected their rough surface scattering characteristics which cause angular dispersion in the received signal. In the LoS applications, the presence of a building can cause shadowing or conversely, provide an alternate ray path to beam form along. Past research works for corner diffraction have focused on cellular applications at 28 and $40 \mathrm{GHz}$, and diffraction from metal and wooden wedges at $60 \mathrm{GHz}$. In addition to the diffraction from building corners, the columns, window frames, etc. on building surfaces are expected to give significant contributions to the received signal. The knowledge of where the relevant scaterers on the surface are, can speedup beam-finding times 
and enhance performance" [6]. Limited work has been done in the $60 \mathrm{GHz}$ band, while little or none has been found in the new $100 \mathrm{MHz}$ bandwidth provided in the $24 \mathrm{GHz}$ band for communications networks. This work serves to fill this gap by investigating the enhanced performance of millimeter $(24 \mathrm{GHz})$ wave in NLoS due to diffraction propagation.

"Diffraction can influence the received power in indoor environments, especially in areas that are heavily shadowed. Though according to [7] , diffraction does not constitute a relevant propagation mechanism already at mm-waves in their investigation with $60 \mathrm{GHz}$, and therefore concluded that, since diffraction losses increase with frequency, this mechanism can be neglected for propagation prediction in the $\mathrm{THz}$ range but the results of this work shows that diffraction propagation at $24 \mathrm{GHz}$ enhanced the received power. This as depicted in the results will facilitate the effective and optimum utilization of such network in the office as well as nlos environments.

The advantage of a commercially-available $24 \mathrm{GHz}$ point-to-point link wireless system (Ubiquiti airFiber) was utilized in this work, this wireless system uses either horn or parabolic dish antennas [8][9][10]. This system featured both multiple-input/multiple-output (MIMO) technologies, dynamically variable signal constellations together with adaptive time/frequency multiplexing, while both frequency and time division multiplexing are used in hybrid form: HDD; the combination of the best features of both TDD and FDD (e.g. interference-reduction and flexible band-planning) [11][12] enhances the realization of the specified $1.4 \mathrm{Gbps}$ delivery as established by the results of the real-time application measurements conducted earlier. It is well-known that mm-wave links have intrinsically smaller Fresnel zones, as a guide, $60 \%$ of this zone should be unobstructed [13], otherwise it is deemed as NLoS as in the indoor environment. $24 \mathrm{GHz}$ point-to-point link used in these experiments had a narrow beam width of 3.5 degrees hence an obstruction smaller than the wavelength of the transmitted signal would cause scattering while a rough surface would diffuse the radio signal in all directions [14].

\section{Methodology and Experimental Setup}

The experimental set up shown in Figure 1 consists of the $24 \mathrm{GHz}$ point-to-point link with maximum transmitter output power of $20 \mathrm{dBm}$. It has delivery capacity of $1.4 \mathrm{Gbps}$ using the HDD in bidirectional mode at 6X64 QAM modulations scheme (highest) and is backward compatible to lower modulation scheme of QPSK through the automatic rate adaptation to accommodate low signal transmission. The feature enables a link pair to sustain up to $142.5 \mathrm{~dB}$ path loss when switched to basic QPSK modulation mode. Full duplex transmission is used with slight different carrier frequency of 24.1 and $24.2 \mathrm{GHz}$; a bandwidth of $100 \mathrm{MHz}$ [12]. The transmitting and the receiving terminals have an antenna gain of $33 \mathrm{dBi}$ each. For the experimental measurement, both antennas were mounted on tripods $1.7 \mathrm{~m}$ above the floor level, and connected to PCs for signal transmission monitoring. A board was arranged in between the links to provide an edge/obstruction to allow diffraction propagation from the source to the receiving terminal. The diagrammatical representation of the transmission links is shown in Figure 2. 


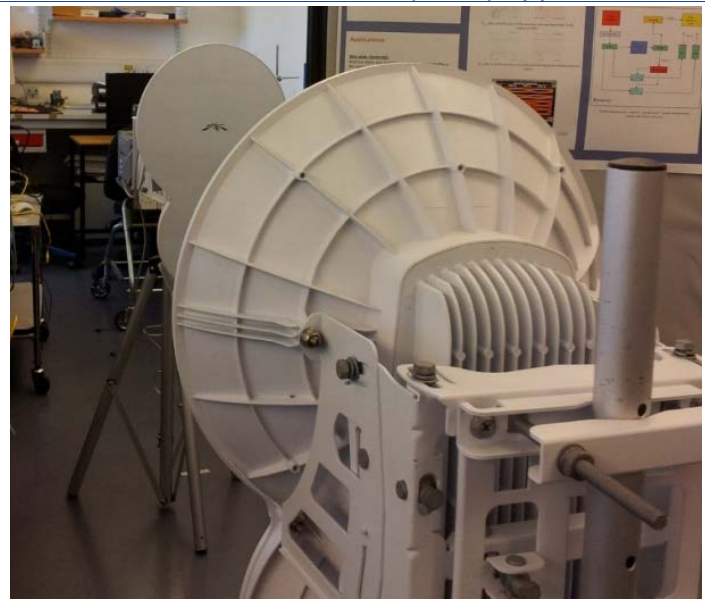

Figure 1: Diffraction Propagation experimental Set Up

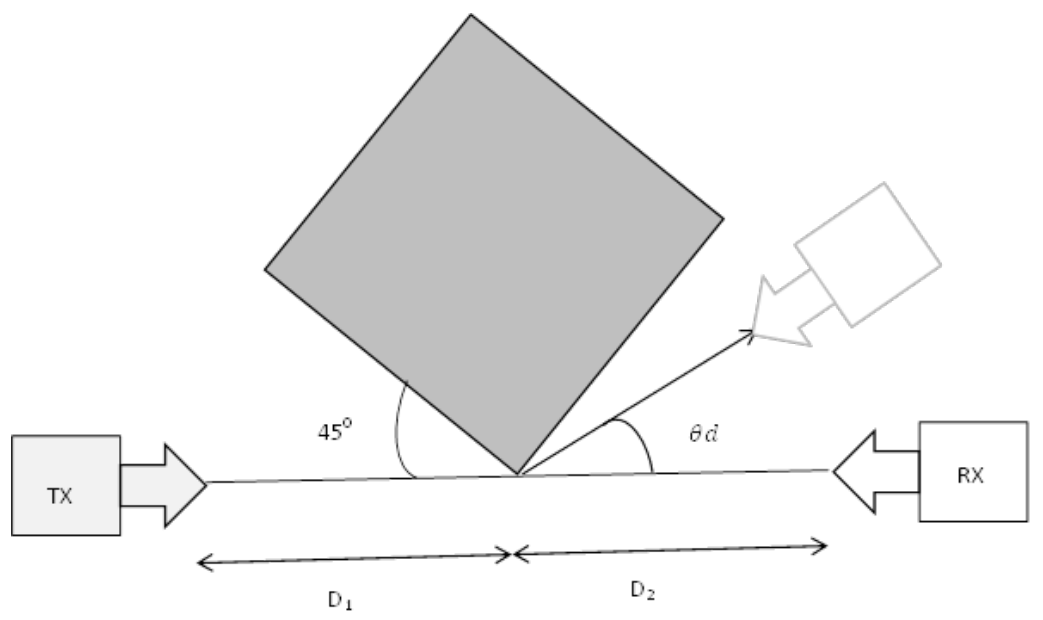

Figure 2: Corner edge propagation measurement Setup

\section{Results and discussion}

The 3.5 degrees bore sight of $24 \mathrm{GHz}$ transceiver is significant. The divergence of the Radiation Pattern is confined within a narrow spread even in the clustered and potentially diffractive scenario where this experiment was conducted. This aided the transmitted signal to focus and concentrated the transmitted power to the receiving terminal with little or no waste along the signal path, hence impressive results were achieved with increase in the diffraction propagation angles. The terminals were initially set at LoS to each other and latter, the edge of a board was arranged to provide obstruction of signal on the transmission path. The receiver links was rotated around the obstruction edge at 5 degree step for reception until total fading was achieved at 45 degrees shadowing created by the board. The curves of the signal attenuation and the different angles of diffraction are presented in figures 3-5. Also on the curve of the diffraction propagation loss, is the free space path loss of the device for comparison purpose. 


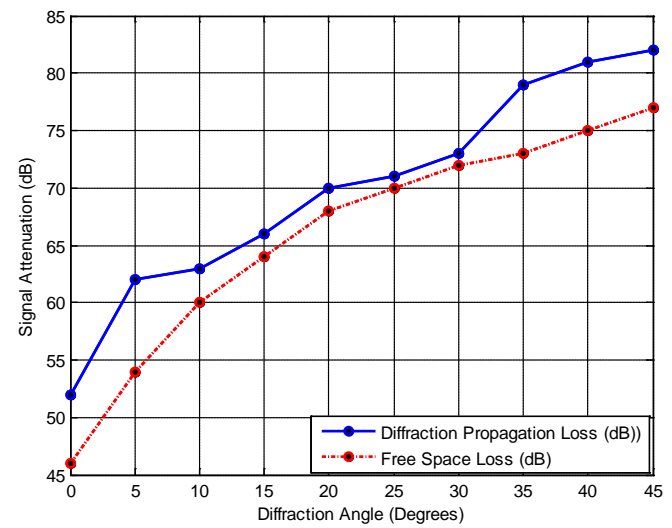

Figure 3: Signal Attenuation at different angles of diffraction and Free Space path loss

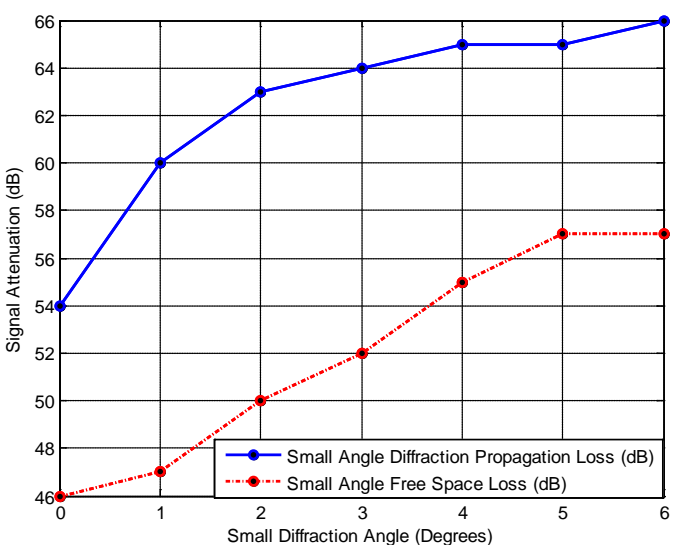

Fig 4: Signal Attenuation at small angles of diffraction Propagation and Free space Path loss

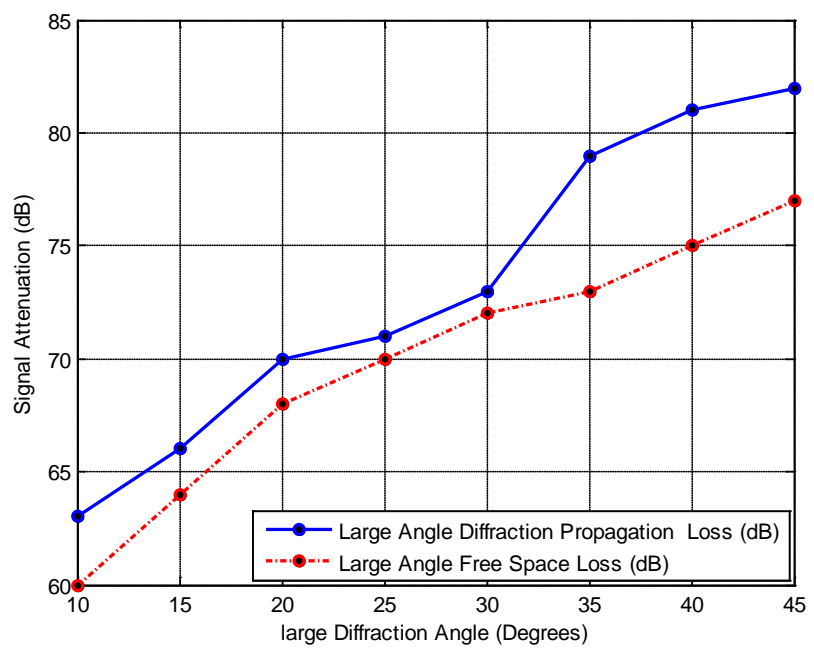

Figure 5: Signal Attenuation at Large angles of diffraction Propagation and Free space path loss

\section{Conclusion}

The enhanced signal transmission in an obstructed environment as revealed by the experimental results of this work shows that the integration of directional antennas in millimeter wave WLAN systems enhances the system performance by increasing the capacity and extending the angular range of reception. In out experiments interference was mitigated and multipath effects were equalized to achieve high data rate at reasonably diffraction angles. It is can be concluded that wireless networks can now compare favorably with their wired counterparts for gigabit data rate delivery required by the numerous consumer applications in the office and obstructed environments, while security is enhanced due to the antenna directivity, focusing the signal transmission with increased gain while suppressing interference towards the targeted user. The tremendous advantages in this newly opened frequency spectrum at 24Ghz can be tapped into for the realization of fast and seamless high throughput transmission needed for the fast growing ubiquitous wireless and internet applications. 


\section{REFERENCES}

[1]. O.J Femi-Jemilohun and S. D. Walker, "Pathloss Prediction Models for Corridor Propagation at 24GHz," Transactions on Networks and Communications, vol. 2, pp. 84-94, 2014.

[2]. O.J Femi-Jemilohun, T. Quinlan, S Barc, and S.D Walker, "An Experimental Investigation into GbE Wireless data Communication at $24 \mathrm{GHz}$ in Non-Line-of-Sight and Multi-Path Rich environments," Antenna and Wireless Propagation Letters IEEE, vol. 13, pp. 1219-1222, 2014.

[3]. M. Jacob, S. Priebe, R. Dickhoff, T. Kleine-Ostmann, T. Schrader, and T. Kurner, "Diffraction in $\mathrm{mm}$ and sub-mm Wave Indoor Propagation Channels," Microwave Theory and Techniques, IEEE Transactions on, vol. 60, pp. 833-844, 2012.

[4]. P. A. Tenerelli, "Diffraction by Building Corners at $28 \mathrm{Ghz}$ : Measurements and Modeling," Citeseer, 1998.

[5]. P.F.M Smulders "Statistical Characterisation of $60 \mathrm{GHz}$ Indoor Radio Channells" IEEE Transaction of Antenna and Propagatio, vol 57, 2009.

[6]. J. Medbo, etal "Channel Modelling for the Fifth Generation Mobile Communications "

[7]. H. D. Schotten and M. A. Uusitalo (Editors), "Intermediate description of the spectrum needs and usage principles," Deliverable D5.1, V1.0, ICT-317669, METIS project, 30th August 2013.

[8]. S. Kavanagh, "An introduction to 24GHz," Kitohener Waterloo Amateur Radio Club, 2001.

[9]. A. C. T. Workings, "Hardware and solution for the broadband wireless industry," Altius Communication Technology Workings Electronic Article, 2013.

[10]. J. Laskar, S. Pinel, D. Dawn, S. Sarkar, B. Perumana, and P. Sen, "The next wireless wave is a millimeter wave," Microwave Journal, vol. 50, no. 8, p. 22, 2007.

[11]. S. Yun, S. Park, Y. Lee, D. Park, Y. Kim, K. Kim, and C. G. Kang, "Hybrid division duplex system for next-generation cellular services," Vehicular Technology, IEEE Transactions on Vehicular Technology, vol. 56, no. 5, pp. 3040-3059, 2007.

[12]. Y. J. Sang, J. M. Park, S.-L. Kim, and K. S. Kim, “An overlaid hybrid-division duplex OFDMA system with multihop transmission.," ETRI Journal, vol. 33, no. 4, 2011.

[13]. Digi, “Application note xst-an010a,” Digi, p. 6, 2012.

[14]. J. Zyren and A. Petrick, "Tutorial on basic link budget analysis," Application Note AN9804, Harris Semiconductor, 1998. 